\title{
The Role of Myocardin in Platelet-derived Growth Factor-BB-induced Phenotypic Switching of Vascular Smooth Muscle Cells
}

\author{
Ying Yuan ${ }^{1,}$, , Tiefeng Song ${ }^{1, b}$, Huiqin Wang ${ }^{1, c}$, Liwen Huang ${ }^{1, d}$, Nan Wang ${ }^{1, e}$ \\ ${ }^{1}$ A Key Laboratory of Industrial Microbiology, Ministry of Education and Tianjin City, College of Bio- \\ technology, Tianjin University of Science and Technology, People's Republic of China \\ ababybani@yeah.net, bsongtiefeng1025@126.com, ‘862005325@qq.com, dhlwjessica@163.com, \\ ewn929@tust.edu.cn
}

Keywords: Vascular smooth muscle cells · Proliferative phenotype · Myocardin · PDGF-BB Abstract. In recent years, numerous studies confirm that platelet-derived growth factor-BB (PDGF$\mathrm{BB}$ ) is involved in the development of atherosclerosis and the regulation of human aorta VSMC (HA-VSMC) proliferation. Myocardin is an essential transcription factor that can activate the transcription of some smooth muscle marker genes. Our results showed that HA-VSMCs treated with PDGF-BB exhibited morphological changes from spindle-shaped to polygonal shape. The expression of proliferation marker gene CyclinD1, osteopontin (OPN), proliferating cell nuclear antigen (PCNA) were increased in PDGF-BB-treated HA-VSMCs. 5-ethynyl-2'-deoxyuridine incorporation assay (EdU) and 3-(4,5-dimethylthiazol-2-yl)-2,5-diphenyltetrazolium bromide (MTT) assay confirmed that cell viability and proliferative ability were increased significantly in PDGF-BB treated HA-SMCs. Next, the expression of transcription factor myocardin was decreased in PDGF-BBtreated HA-VSMCs. These results preliminarily demonstrated the role of myocardin in phenotypic switching of PDGF-BB-induced HA-VSMCs.

\section{Introduction}

Plasticity of human aorta vascular smooth muscle cells (HA-VSMCs) plays a central role in the onset and progression of proliferative vascular diseases. In adult tissue, HA-VSMCs exist in a physiological contractile-quiescent phenotype, which is defined by lack of the ability of proliferation and migration, while high expression of contractile marker proteins [1]. After injury to the vessel, VSMCs shift from a contractile phenotype to a pathological synthetic phenotype, associated with increased proliferation, migration and matrix secretion [2]. It has been demonstrated that plateletderived growth factor-BB (PDGF-BB) is a critical mediator of HA-VSMCs phenotypic switch [3]. Myocardin, the master regulator of smooth muscle cell differentiation, induces expression of smooth muscle marker genes through its association with serum response factor (SRF), which binds to the CArG box in the promoter [4]. Therefore, the aim of this study is to establish PDGFBB-induced proliferation model of HA-SMCs and preliminarily investigate the role of transcription factor myocardin on phenotypic switching process of HA-VSMCs.

\section{Materials and methods}

2.1 Cell culture. Human aortic vascular smooth muscle cells were purchased from the Type Culture Collection of the Chinese Academy of Sciences, Shanghai, China. HA-VSMC cells were cultured in dulbecco's minimum essential medium-high glucose (Gibco by life technologies, USA) containing $10 \%$ fetal bovine serum (Sijiqing,China), penicillin $(100 \mathrm{U} / \mathrm{mL})$ and streptomycin $(100 \mathrm{U} / \mathrm{mL})$, at $37{ }^{\circ} \mathrm{C}$ in humified air with $5 \% \mathrm{CO}_{2}$.

2.2 Real-time RT-PCR. Total RNA was extracted from cells using Trizol (Solarbio, China) according to the manufacturer's protocol, and then reverse-transcription was performed using M-MLV reverse transcriptase (Promega, USA) according to the manufacturer's instructions. Real-time quantitative PCR assay was carried out with SybrGreen qPCR Mastermix (DBI, China). The PCR primer sequences are as follows: GAPDH: forward-5'ATTCAACGGCACAGTCAAGG3', reverse5'GCAGAAGGGGCGGAGATGA3'; Myocardin: forward-5'AGTAAGAACCGCCACAAA3', reverse-5'GAGCATAGGCAGAGTCCA3';

CyclinD1:

forward- 
5'GCTGTGCATCTACACCGACAACTC', reverse-5'TTGCGGATGATCTGTTTGTTCTCCT'; PCNA: forward-5'GGCTCCATCCTCAAGAAGGTGTT', reverse5'CGTTATCTTCGGCCCTTAGTGTA'; OPN: forward-5'AGTACCCTGATGCTACAGACGAG', reverse-5'TCGTTTCATAACTGTCCTTCCCAC'.

2.3 Wesltern blotting. Western Blotting was performed as described previously [5]. Equal amount of total protein was loaded on $12 \%$ sodium dodecylsulfate polyacrylamide gel. Then samples were transferred to NC membranes and blocked for $60 \mathrm{~min}$ at RT in 5\% skim milk powder (wt/vol) in phosphate buffer saline (PBS). The membranes were immunoblotted with mouse anti-rat $\beta$-actin (Santa Cruz), rabbit anti-rat Myocardin (Sigma) or rabbit anti-rat Cyclin-D1 (Santa Cruz) antibodies overnight at $4^{\circ} \mathrm{C}$, and then incubated with IRDyeTM- 800 conjugated anti-rabbit secondary antibodies (Li-COR) for $30 \mathrm{~min}$ at RT. The blots were scanned by an infrared imaging system (Odyssey, LICOR).

2.4 MTT assay. HA-VSMCs were cultured to $60 \%$ confluence. Cell viability was examined by MTT assay. Cells were seeded in 96-well culture plates at a density of 4000 cells/well and incubated with $0.5 \mathrm{mg} / \mathrm{mL}$ MTT in the last $4 \mathrm{~h}$ of the culture period at $37^{\circ} \mathrm{C}$. The medium was replaced with $100 \mu \mathrm{L}$ DMSO and the plate was gently rotated on a linear and orbital shaker for $5 \mathrm{~min}$ to completely dissolve the precipitation. An automatic microplate reader was used to detect the absorbance at $490 \mathrm{~nm}$.

2.5 EDU assay. EdU (5-ethynyl-2'-deoxyuridine) incorporation assay was performed to further investigate the impact of PDGF-BB on the proliferation of human ASMCs using the Cell-Light TM EdU imaging detecting kit. All of the EdU incorporation experiments were performed according to the kit's manufacturer's protocol. The percentage of EdU-positive nuclei to total nuclei was viewed as the proliferation rate of human ASMCs in four random high-power fields per well.

\section{Results and discussions}

3.1 The mRNA level of the proliferative markers were increased in PDGF-BB-treated HAVSMCs. After 24h of starvation with serum-free medium, HA-VSMCs were incubated with 20, 40, $60 \mathrm{ng} / \mathrm{mL}$ PDGF-BB for 12h, 24h, 48h, 72h. As shown in Fig 1A, administering 20, 40, $60 \mathrm{ng} / \mathrm{mL}$ of PDGF-BB into HA-VSMCs resulted in the upregulation of proliferative marker gene CyclinD1, PCNA and OPN expression. Next, HA-VSMCs were cultured in medium containing $40 \mathrm{ng} / \mathrm{mL}$ of PDGF-BB for 24, 48, 72 hours. Stimulation of HA-VSMCs with $40 \mathrm{ng} / \mathrm{mL}$ PDGF-BB for $24 \mathrm{~h}$ and $48 \mathrm{~h}$ caused significant increase in the expression of proliferative marker genes (Fig. 1B).

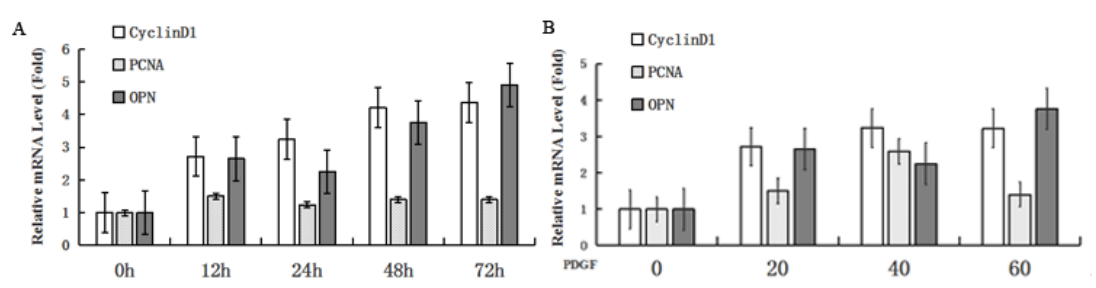

Fig. 1. The expression of CyclinD1, OPN and PCNA in PDGF-BB-stimulated HA-VSMCs. (A) Expression of CyclinD1, OPN and PCNA were detected in 20, 40, $60 \mathrm{ng} / \mathrm{mL}$ PDGF-BB-treated HA-VSMCs by Real-time PCR. (B) Expression of CyclinD1, OPN and PCNA were detected in HA-VSMCs treated with $40 \mathrm{ng} / \mathrm{mL}$ PDGF-BB for 24, 48, 72 hours by Real-time PCR.

3.2 PDGF-BB treatment resulted in the increase of the proliferative marker CyclinD1 expression. HA-VSMCs were cultured in medium containing $40 \mathrm{ng} / \mathrm{mL}$ PDGF-BB for $48 \mathrm{~h}$ and then the protein level of CyclinD1 was detected by western blotting. As shown in Fig. 2, the expression of proliferative marker gene CyclinD1 was increased in PDGF-BB-treated HA-VSMCs. Therefore, these data preliminarily suggested that the HA-VSMCs proliferation model induced by PDGF-BB was successfully established. 


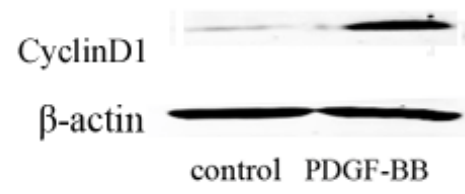

Fig. 2 Expression of CyclinD1 was detected in PDGF-BB-treated HA-VSMCs by Western blotting

3.3 PDGF-BB induced HA-VSMC proliferation. After HA-VSMCs were stimulated with PDGF-BB, cells exhibited morphological changes from spindle-shaped to polygonal shape, exhibited proliferation phenotype forms (Fig. 3A).To investigate the effect of PDGF-BB in the proliferation of vascular smooth muscle cells, HA-VSMCs were treated with $40 \mathrm{ng} / \mathrm{mL}$ PDGF-BB for $24 \mathrm{~h}$ and then EdU staining and MTT assay was performed. Treatment with $40 \mathrm{ng} / \mathrm{mL}$ PDGF-BB induced proliferation of VSMCs in comparison with non-stimulated cells (Fig. 3B and C).
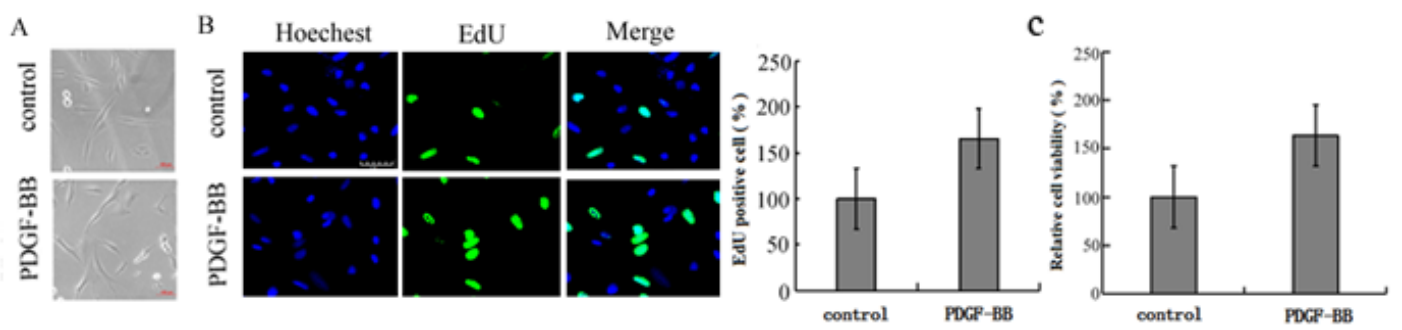

Fig. 3 The morphological changes and EdU staining of HA-VSMCs stimulated by PDGF-BB. (A) The morphological changes of PDGF-BB-treated HA-VSMCs. (B) EdU staining of PDGF-BBtreated HA-VSMCs. (C) The proliferation of PDGF-BB-treated HA-VSMCs by MTT assay.

3.4 Transcription factor myocardin could be involved in smooth muscle cell phenotype transformation. To investigate the expression of myocardin in PDGF-BB-induced proliferation model, HA-VSMCs were treated with $40 \mathrm{ng} / \mathrm{ml}$ PDGF-BB for $24 \mathrm{~h}$ and then the expression of myocardin was tested by RT-PCR and western blotting. As shown in Fig. 5, the expression of myocardin was decreased in PDGF-BB-induced proliferation model. Therefore, these data suggested that the transcription factor myocardin was involved in smooth muscle cell phenotype transformation.

A

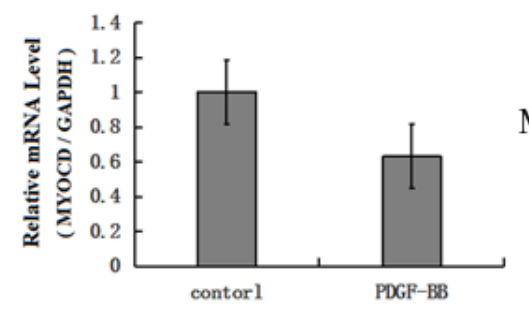

B

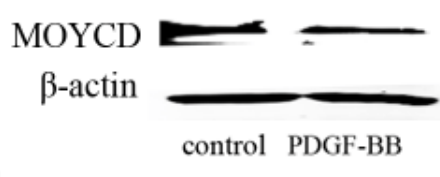

Fig. 4 Expression of myocardin was decreased in PDGF-BB-treated HA-VSMCs by real-time PCR (A) and western blotting (B).

\section{Conclusions}

Switching of vascular smooth muscle cells (VSMCs) from a contractile phenotype to a pathological synthetic state is known to occur in atherosclerosis or vascular restenosis. Many studies have reported that (PI3K)/AKT signaling pathway is implicated in the PDGF-BB-induced proliferation, migration and the changes of cytoskeleton of HA-VSMCs $[6,7]$, which are fundamental features involved in the phenotype modulation [8]. Our results showed that PDGF-BB could promote the proliferation of SMCs. PDGF-BB treatment led to the upregulation of the proliferative marker expression confirmed by real-time PCR and western blotting, the increase of cellular viability confirmed by MTT assay, and the increase of cellular proliferation confirmed by EdU staining.

Myocardin is a key regulator of SMC differentiation. We tested the expression of transcription factor myocardin by RT-PCR and western blotting in HA-VSMCs. Our results demonstrated that the expression of myocardin was enhanced in PDGF-BB-treated HA-VSMCs. These results prelim- 
inarily suggest that myocardin plays an important role in phenotypic switching of HA-VSMCs.

\section{Acknowledgments}

This work was financially supported by the National Natural Science Foundation of China (31171303, 31301073 and 31470816).

\section{References}

[1] G.K. Owens , M.S. Kumar and B.R. Wamhoff. Physiological reviews. 84 (2004), p. 767-801

[2] M.L. Bochaton-Piallat, G. Gabbiani. Handbook of experimental pharmacology. 170 ( 2005), p.645-63

[3] S. Chen, B. Liu and D. Kong. PLoS One. 10 (2015), p. 1-15

[4] T.E. Callis, K. Pandya and H.Y. Seok . J Clin Invest. 119 (2009), p. 2772-86

[5] W. Liu, X. Zen, J. Wang, Z. Wang, X. Yan, Y. Wang and H. Wang. Exp Cell Res. 336 (2015), p. 204-10

[6] E.A. Goncharova, A.J. Ammit, C. Irani, R.G. Carroll and A.J. Eszterhas. American journal of physiology Lung cellular and molecular physiology. 283 (2002), p. 354-63

[7] K.H. Choi, J.E. Kim, N.R. Song, J.E. Son, M.K. Hwang, S. Byun. Cardiovascular research. 85(2010), p. 836-44

[8] M. Mandegar, Y.C. Fung, W. Huang, C.V. Remillard, L.J. Rubin, J.X. Yuan Microvascular research. 68 (2004), p. 75-103 